Revue d'histoire de l'Amérique française

REYUE D.HISTOIRE DE L'AMÉRIQUE FRANÇAISE

GROUPE DE RECHERCHE EN HISTOIRE RÉGIONALE, Bibliographie d'histoire des Cantons de l'Est. Département d'histoire, Université de Sherbrooke, 1975. 120 p. Index. \$2.00

\title{
René Hardy
}

Volume 30, numéro 3, décembre 1976

URI : https://id.erudit.org/iderudit/303549ar

DOI : https://doi.org/10.7202/303549ar

Aller au sommaire du numéro

Éditeur(s)

Institut d'histoire de l'Amérique française

\section{ISSN}

0035-2357 (imprimé)

1492-1383 (numérique)

Découvrir la revue

Citer ce compte rendu

Hardy, R. (1976). Compte rendu de [GROUPE DE RECHERCHE EN HISTOIRE RÉGIONALE, Bibliographie d'histoire des Cantons de l'Est. Département d'histoire, Université de Sherbrooke, 1975. 120 p. Index. \$2.00]. Revue d'histoire de l'Amérique française, 30(3), 422-424. https://doi.org/10.7202/303549ar d'utilisation que vous pouvez consulter en ligne. 
Groupe DE RECHERCHE EN hISTOIRE RÉGIONALE, Bibliographie d'histoire des Cantons de l'Est. Département d'histoire, Université de Sherbrooke, 1975, 120 p., index. $\$ 2.00$

Cette bibliographie d'histoire des Cantons de l'Est est le résultat d'un travail de deux ans de recherche fait en collaboration par des professeurs et des étudiants diplômés du Département d'Histoire de l'Université de Sherbrooke. J'acquiesce à l'opinion d'Andrée Désilets, émise en avantpropos, qu'il convenait que le premier ouvrage de ce groupe de recherche en histoire régionale fût un inventaire des imprimés. N'est-ce pas un moyen sûr, en dévoilant ainsi les divers aspects de l'objet d'étude, de multiplier les questions et de susciter la réflexion scientifique?

L'ouvrage est constitué d'environ 900 titres choisis parmi les 2,000 références que contiennent les fichiers de leur centre de recherche en histoire régionale. Les chercheurs y trouveront certainement l'essentiel sur le sujet et même beaucoup plus. Mais pour avoir un aperçu, un tant soit peu complet des publications traitant de cette partie du Québec, cet inventaire ne suffira pas, car les auteurs ont choisi, de façon explicite, de ne pas indexer toutes les publications gouvernementales déjà recensées ailleurs et de s'en tenir aux ouvrages relatifs à une bibliographie d'histoire.

Qu'entend-on ici par «bibliographie d'histoire»? Je ne tenterai pas de définir ce que les auteurs ont omis de faire en introduction. Il vaut mieux montrer, à l'aide d'exemples, que cette définition aurait été utile pour fixer le chercheur sur le contenu de la bibliographie et comprendre le plan de classification. Quant au contenu, le lecteur sera d'abord surpris de trou- 
ver dans une bibliographie d'histoire une étude sur la géologie des environs de Marbleton et comprendra difficilement pourquoi les auteurs ont ignoré, entre autres ouvrages, une étude pédologique du comté de Drummond, une étude récente sur la rénovation urbaine ou encore une analyse de la pollution de l'eau. Faut-il l'expliquer par l'unique désir d'être sélectif ou par leur conception de la bibliographie d'histoire? Le plan de classification me semble aussi souffrir des mêmes ambiguïtés. L'ouvrage est divisé en 5 parties: 1/ Instruments de travail ; 2 / Études régionales; 3/ Études locales; 4/ Études thématiques; $5 /$ Sciences connexes. Cette dernière rubrique ne laisse-t-elle pas supposer que les autres - à l'exception de la première - relèvent de l'histoire? Je me demande alors selon quels critères un petit ouvrage sur les structures démographiques et économiques de la région de l'amiante est classé dans la section "Études locales - comté de Mégantic», un dossier sur la démographie estrienne dans «Études thématiques - sociétépopulation", une thèse d'un géographe traitant de l'industrie manufacturière dans "Études thématiques - économie-industrie», une autre thèse d'un géographe sur le commerce d'alimentation de détail dans «Sciences connexes - géographie» et une recherche sur les facteurs régionaux de localisation industrielle dans "Sciences connexes - économie». Bref, les trois premiers titres sont classés dans la section histoire et les deux autres dans les sciences connexes.

Ces exemples me semblent rendre compte des difficultés inhérentes à un plan de classement à la fois thématique et disciplinaire. L'un et l'autre se recoupent et, à la limite, peuvent se dédoubler. Ici, tous les titres inscrits à "Sciences connexes - économie et géographie» pourraient être placées à "Études thématiques - économies" ou à "Études régionales". Quelle conception de la bibliographie d'histoire justifiait donc l'ajout de la section «Sciences connexes»?

J'admets cependant que dans le cas présent, grâce à une bonne table des matières, à un index des noms d'auteurs, à un système de renvois bien élaboré et grâce au volume plutôt restreint de cette bibliographie, les défauts signalés en gênent très peu la consultation. Mais appliqué à un corpus documentaire plus large, ce plan de classification laisserait voir ses inconvénients.

Il faut enfin déplorer que les auteurs n'aient pas jugé bon de définir la région étudiée. Des informations puisées dans la table des matières permettent de déduire que les Cantons de l'Est désignent ici tout le territoire de 1791, plus quelques modifications faites aux limites des comtés frontaliers au $20^{\mathrm{e}}$ siècle. Il ne semble pas que ce territoire soit une région "naturelle» au sens entendu chez les géographes, ni une région économique intégrée, ni une région administrative, la région 05 étant beaucoup plus restreinte. Est-ce une région culturelle? Si j'en juge par les opinions entendues dans les Bois-Francs et les témoignages rapportés par Jean Mercier dans son livre L'Estrie, les habitants de cette région se définissent souvent diffé- 
remment des habitants de la région de Sherbrooke. Est-ce donc essentiellement une appellation historique recouvrant aujourd'hui des réalités régionales diversifiées?

La réflexion sur le sujet est déjà amorcée par le groupe de recherche de Sherbrooke. D'autres groupes se sont aussi constitués ailleurs au Québec. Souhaitons que s'instaure la collaboration à laquelle nous invite cette bibliographie en nous indiquant la profusion des publications et la richesse des études existantes sur cette partie du Québec.

Section d'Histoire

RENÉ HARDY

$U Q T R$ 